\title{
CORRECTIONS
}

\section{Problems of stopping trials early}

This Analysis article by Guyatt and colleagues contains a typographical error (BMJ 2012;344:e3863, doi:10.1136/bmj. e3863) in the second paragraph of the section " $\beta$ blockers in non-cardiac surgery." The odds ratio documented for the "twofold increase in non-fatal strokes" has a 95\% confidence interval of 1.27 to 3.68 (not "27 to 3.68 " as was published).

Cite this as: $B M J$ 2014;348:9319

๑ BMJ Publishing Group Ltd 2014 